\title{
Anticipating incoming events: an impaired cognitive process in schizophrenia
}

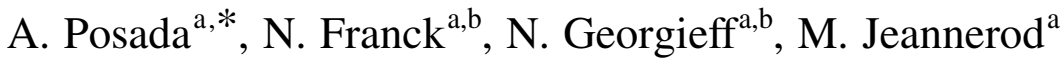 \\ anstitut des Sciences Cognitives, 67 Boulevard Pinel, 69675 Bron, France \\ ${ }^{\mathrm{b}}$ Centre Hospitalier Le Vinatier and EA 3092 (INFL), Université Claude Bernard, Lyon, France
}

Received 10 January 2001; accepted 23 March 2001

\begin{abstract}
Intentions are central to guiding actions to their completion because they generate expectations which precede the realization of a task. This ability to manage time was investigated by using a cognitive task which involves several highly integrated processes: sequential learning, explicit processing, and working memory. In this task, participants are required to explicitly learn a repeating color sequence before receiving an instruction to give an anticipatory motor response concerning the next element. Two types of sequences (temporal and spatial) and three experimental conditions were tested in both a group of normal participants and a group of schizophrenic patients. Schizophrenics were included because their condition is known to alter conscious executive function. Our results showed that schizophrenic patients have a strong deficit in performing anticipation tasks. Although they learned the sequences almost normally, their anticipatory ability was reduced in comparison to normal participants in all the tested conditions. These results expand the notion of a working memory deficit in schizophrenia and bear strong implications for understanding executive disorders observed in such patients. (C) 2001 Elsevier Science B.V. All rights reserved.
\end{abstract}

Keywords: Schizophrenia; Working memory; Behavioral task; Anticipation; Executive functions; Consciousness; Frontal lobe

\section{Introduction}

Actions are intended before being performed, which implies that their goals and

* Corresponding author.

E-mail address: posada@isc.cnrs.fr (A. Posada).

0010-0277/01/\$ - see front matter (C) 2001 Elsevier Science B.V. All rights reserved.

PII: S0010-0277(01)00133-0 
potential consequences are represented by the agent. In this way human behavior is predictive. Although predictive behavior is at work in nearly all human everyday activities, it can also be the object of experimental study in simplified situations. One possible approach consists of monitoring responses of participants in tasks where they are required to anticipate events by using information that is either provided to them repeatedly or acquired explicitly through learning. For example, consider the action of learning a sequence of colors that repeatedly appear in the same order on a computer screen: a participant who knows that the colors appear in a fixed order learns the sequence voluntarily, simply by watching the computer screen and repeating the color names verbally. If, during this process of learning, the participant is instructed to press a specific key each time a given color appears on the screen, the time to press the key sharply decreases after the complete sequence has been learned and verbalized. As a matter of fact, keys are now pressed before the next color is shown. This ability to produce anticipatory responses reflects the elementary process which ultimately allows predictive behavior in natural situations.

The generation of such responses relies on conscious processing of the relevant information. In the case of the above color sequence task, it requires verbal coding of the color names, storage of their ranking order, memorization of the instruction, a decision to activate the response before a color appears and generation of an intention shift in time. These operations are likely to depend on a conscious system controlling sensorimotor representations and memory management, cognitive functions generally grouped under the concept of working memory (see Baddeley, 1998). A failure of this system, for example bad synchronization between memory elements and motor commands, would unavoidably result in a situation where anticipatory responses and predictive behavior would become impossible.

In this paper we consider the case of schizophrenia. Deficient explicit and conscious modalities of processing are dominant characteristics of this pathological condition (Aleman, Hijman, de Haan, \& Kahn, 1999; Fleming et al., 1997; Goldman-Rakic, 1994; Wexler, Stevens, Bowers, Sernyak, \& Goldman-Rakic, 1998; see Kuperberg \& Heckers, 2000, for review). This is also true for cognitive tasks where working memory is likely to be involved, like learning abstract sequences (Dominey \& Georgieff, 1997), management of rules (e.g. in the Wisconsin card sorting test; Laws, 1999), attention tasks (Bernard, Lancon, \& Bougerol, 1997), processing of context (Servan-Schreiber, Cohen, \& Steingard, 1996), recognition memory (Danion, Rizzo, \& Bruant, 1999) or semantic processing (Kuperberg, McGuire, \& David, 1998). Also they do not benefit from regular and predictable preparatory intervals in simple reaction time tasks (Nuechterlein \& Dawson, 1984). Finally, schizophrenic patients show strong deficits in working memory tasks, especially with tasks requiring the stability of a representation like the Stroop test (Grapperon $\&$ Delage, 1999). In these patients, working memory deficits have also been evaluated using the oculomotor 'anti-saccades' paradigm, which requires that subjects can hold 'on-line' the position of a target, suppress a response and generate an inverse movement (Crawford, Haeger, Kennard, Reveley, \& Henderson, 1995; Walker, Husain, Hodgson, Harrison, \& Kennard, 1998). Alternatively the delayed paradigm, which requires the generation of a saccade after the target disappears, can 
be used (Park, Holzman, \& Goldman-Rakic, 1995). Although in these oculomotor tasks some anticipatory behavior can be observed, the responses are not explicitly produced and are not based on previously learned knowledge. In this study, we propose to evaluate the functional role of the conscious working memory system in generating motor responses before an external event occurs.

The ability of schizophrenic patients to anticipate forthcoming events was therefore studied in an explicitly learned task, using the color sequence task mentioned above. A group of patients and a group of matched control participants were included in two experiments with different types of sequences: in one (the Temporal experiment), stimuli were presented as a simple temporal sequence at the same spatial locus; in the other (the Spatial experiment) the items of the sequences were spatially distributed in a manner that followed geometrical shapes. In both experiments, the participants' abilities to anticipate were first tested in a condition where they knew the existence of the sequence and had to learn it. Subsequently, they were tested in two more conditions (Easy and Difficult) where they explicitly received the sequence from the experimenter. Although patients were found to be able to acquire the sequence almost normally, they proved to be impaired in using their explicit knowledge to produce anticipatory responses.

\section{Methods}

\subsection{Participants}

The study was conducted with a group of 20 chronic schizophrenic patients who met DSM-IV criteria. They were recruited in a local psychiatric hospital (Le Vinatier). Exclusion criteria were neurological or medical illness which could affect the nervous system, alcohol or drugs abuse, substance dependence, and treatments with lithium, benzodiazepines or antidepressants.

The patients (six women and 14 men) had a mean age of $36.4 \pm 7.9$ years and a mean educational level of $10.8 \pm 2.2$ years. They were classified as paranoid $(n=10)$, undifferentiated $(n=5)$, residual $(n=4)$ and disorganized $(n=1)$ according to DSM-IV criteria. All patients were clinically stable at the time of evaluation and testing. Schizophrenic symptoms were assessed by the Scale for Assessment of Positive Symptoms (SAPS) (Andreasen, 1984) and the Scale for Assessment of Negative Symptoms (SANS) (Andreasen, 1983). The total SAPS score was $28.8 \pm 15.2$, and the total SANS score was $36.5 \pm 22.4$ (see Table 1).

A control group of 20 participants (nine women and $11 \mathrm{men}$ ) also participated in the study. They were matched for age $(33.9 \pm 11.7$ years $)$ and educational level $(12.3 \pm 3.5$ years). They were recruited from the hospital community and colleagues. Exclusion criteria for controls was the same as for patients (plus we included any current or past psychiatric illness). All participants gave their informed consent for participating in the experiment. 


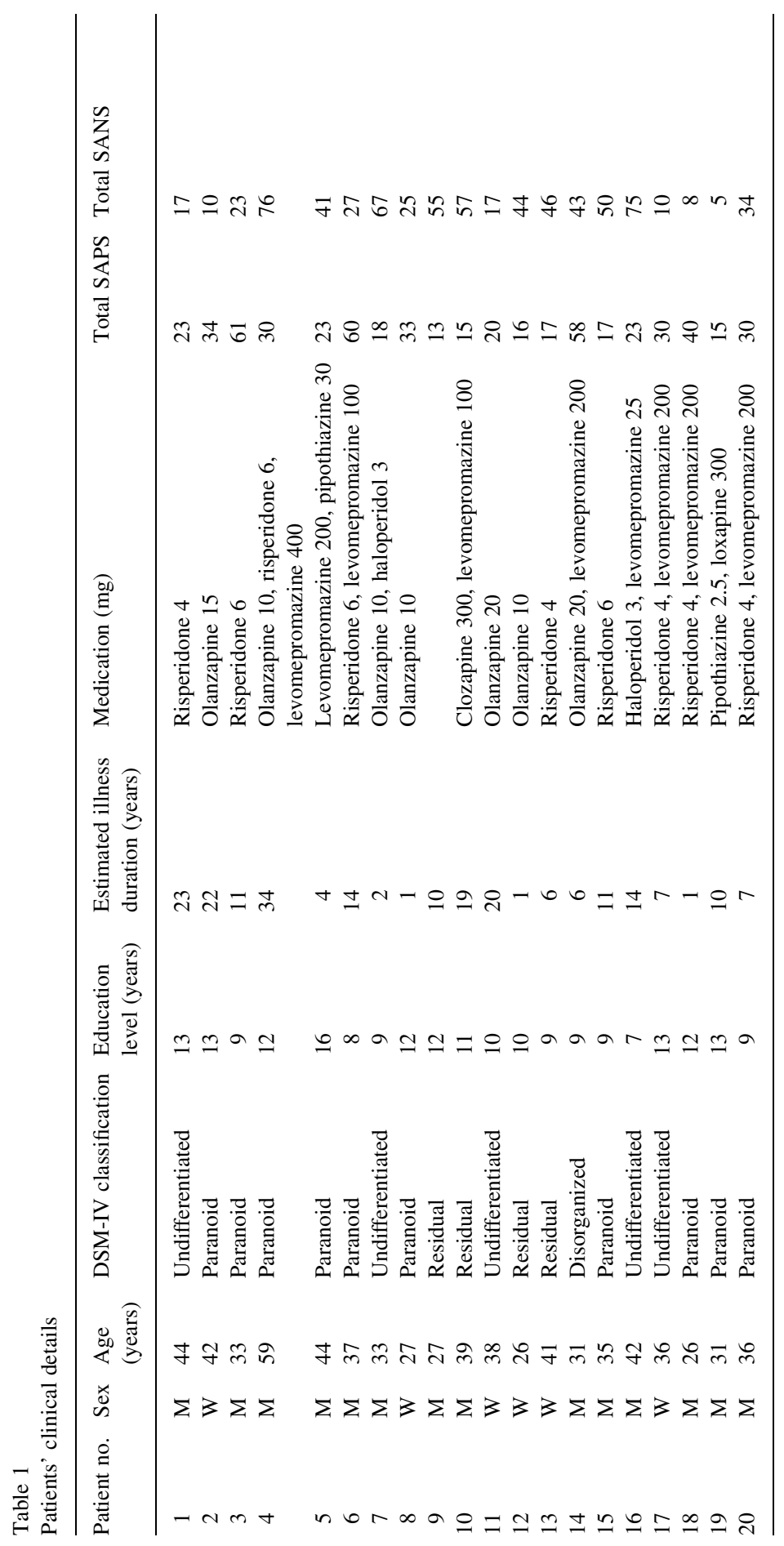




\subsection{Apparatus}

Participants were seated in front of the screen of a notebook PC computer. Sequences of colored (yellow, green or blue) rectangles were displayed on the screen. Whenever a rectangle appeared, participants were instructed to push the corresponding color button on a button box. Response time (RT) was recorded. We used Expe6 (Pallier, Dupoux, \& Jeannin, 1997), a programming tool for the design of neuropsychological protocols, to implement the task.

\subsection{Experimental procedure}

Two experiments were run, one involving purely temporal sequences of rectangles (Temporal experiment), and the other involving temporal sequences where the rectangles appeared at different locations (Spatial experiment).

In the Temporal experiment, the color rectangles $(4 \times 2 \mathrm{~cm})$ appeared in the middle of the screen. For each trial, feedback was given after the participant's response. After a correct response, the word correct appeared on the screen for $1000 \mathrm{~ms}$; then the color rectangle and the feedback disappeared and the screen darkened for a period of $1500 \mathrm{~ms}$ before the next element of the sequence appeared. After an erroneous response, the word error appeared on the screen for $1400 \mathrm{~ms}$; then the color rectangle and the feedback disappeared and the screen darkened for a period of $1000 \mathrm{~ms}$ before the same element reappeared until the participant responded correctly.

In the Spatial experiment, a circle composed by 12 rectangles $(2 \times 1 \mathrm{~cm})$ was displayed on the screen. Each rectangle took on one of the three colors (yellow, green or blue). The colors were randomly distributed over the circle, and the distribution was changed between each block of trials. For each trial, one of the rectangles was increased in size $(4 \times 2 \mathrm{~cm})$. This size enlargement was a cue to the participant to respond by pushing the corresponding color button. Feedback was given after each trial as in the previous experiment. Then, the rectangle returned to normal size and the next rectangle in the sequence increased in size after $1500 \mathrm{~ms}$.

\subsection{Experimental conditions}

Each experiment involved three conditions, each divided into several blocks. In each block the sequence was repeated to yield 12-21 trials per block.

In the first condition (Sequence learning), the participant was informed that the colors were distributed in a fixed sequence, and was instructed to try to disclose the sequence and to report it to the experimenter, either verbally (in the Temporal experiment) or by showing the rectangles on a clock face (in the Spatial experiment). The number of blocks needed to disclose the sequence was measured. For the Temporal experiment, sequences involved four colors (e.g. Green, Blue, Yellow, Blue) and were repeated three times. In the Spatial experiment, sequences involved six clock hours (e.g. 12, 4, 8, 6, 10, 2) and were repeated two times (Fig. 1a).

Whenever a participant could not find the sequence after seven blocks, he/she was helped by the experimenter until he/she discovered the sequence. After the sequence 


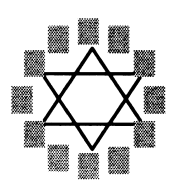

a

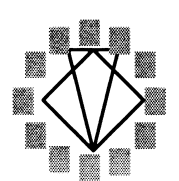

b

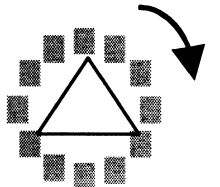

c

Fig. 1. Sequences used in Spatial experiment. (a) Learning condition (12-4-8-6-10-2); (b) Easy sequence (12-3-6-9-6-11-1); (c) Difficult sequence (12-4-8-1-5-9-2-6-10-3-7-11-4-8-12-5-9-1-6-10-2).

was learned, three blocks were presented, where the participant received the instruction to try to push the correct color button before the rectangle appeared, in other words to anticipate the next element of the sequence. Finally, in the last block, a random sequence (12 trials) was generated and the participants were instructed to respond as quickly as possible.

In the second and third conditions (Anticipation conditions), the repetitive sequences (Easy and Difficult, respectively) were explicitly given to the participant before the experiment. The participant received the instruction to try to push the correct buttons before the next element of the sequence appeared on the screen (anticipation). Seven blocks were run, followed by a final block where a random sequence was presented. For the Temporal experiment, the Easy sequences were composed of four colors (the same but inverted first condition sequence) and were repeated three times per block as in the first condition; the Difficult sequences were composed of eight colors (addition of the first and second condition sequences) and were presented two times per block. For the Spatial experiment, the Easy sequences were composed of seven positions and were repeated two times (Fig. 1b); the Difficult sequences were composed of 21 positions per block and were repeated once (Fig. 1c). The number of trials in the final, random sequence, block was adjusted so as to match the number of trials in the different sequences.

\subsection{Data analysis}

The analysis focused on the anticipatory RT data for the repeated sequences (with or without anticipation) and the random sequences (control). The first trial was eliminated in each block. Similarly, all error responses plus the correct responses that followed error responses were eliminated. Due to this procedure, only correct RTs representative of the anticipation capacity of participants were retained. For the three conditions, a percentage of anticipation was computed with the mean of all the blocks' data for the repeating sequences:

percentage of anticipation $=100\left(1-\frac{\text { mean }(\mathrm{RT} \text { repetitive sequence })}{\text { mean }(\mathrm{RT} \text { random sequence })}\right)$

The percentage of anticipation was also computed using the mean of the best 
anticipation' block. A value of $100 \%$ means that the participant anticipated all the trials; 0\% means that the participant never anticipated. In the latter case, the RTs should be equal to the RTs collected during the random sequence presentation.

The number of errors was also scored in order to get an estimate of the participants' capacity to associate a colored stimulus with a colored button.

\section{Results}

The variable used for the measures was RT, i.e. the time to generate a key press response when a given color was presented. RT was maximal when participants simply reacted to the appearance of a color on the screen. It progressively decreased as participants learned the sequence and responded in advance with respect to the presentation. The value of $\mathrm{RT}=0$ was considered for all the cases where the response was generated at the time of the presentation or before the presentation was made.

\subsection{Errors}

The rate of errors in the different experiments and conditions was analyzed. No significant differences between the two groups (control and schizophrenics) ( $t$-test, bi-varied) were found. In both groups the number of errors remained small (Fig. 2).

\subsection{Learning condition}

In the first condition of each experiment (Temporal and Spatial), the number of blocks necessary to disclose and to explicitly report the sequence was determined. Participants who failed to disclose the sequence after seven blocks are reported as "failures". Schizophrenic participants needed $3.4 \pm 1.6$ blocks ( $n=14$; six failures $)$

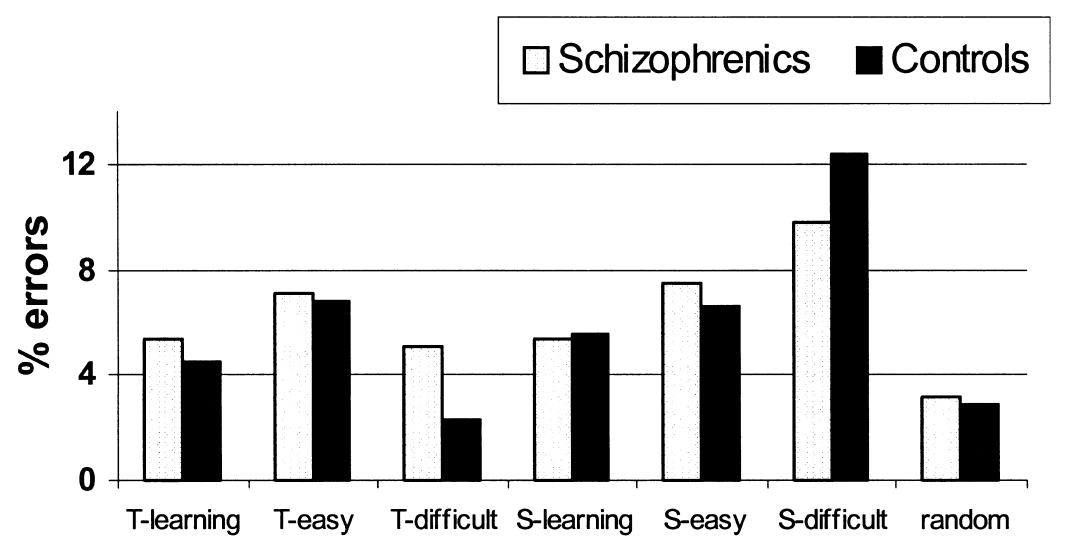

Fig. 2. Percentage of errors in the three conditions (learning, anticipation easy and anticipation difficult) of the two experiments ( $T=$ Temporal experiment and $S=$ Spatial experiment) for Schizophrenics and Controls. We have separated the random sequences (random). 
to find the temporal sequence and $3.2 \pm 1.2$ blocks to find the spatial one ( $n=15$; five failures). Controls needed $2.6 \pm 1.61$ blocks ( $n=18$; two failures) to find the temporal sequence and $2.1 \pm 0.64$ blocks to find the spatial one $(n=18$; two failures). No significant difference was found in the number of blocks between groups for the temporal sequences ( $t$-test, $P=0.2)$. For the spatial sequences, the difference was significant ( $t$-test, $P=0.003)$.

The RT changes through the blocks of the sequence learning condition in the Temporal and Spatial experiments for schizophrenic and control participants are shown in Fig. 3. Blocks 1-3 represent the last three blocks before participants disclosed the sequence. No significant difference in RTs between groups was found for either Temporal or Spatial experiments (ANOVA, Table 2). Note that in block 1, when participants completely ignored the sequence, the two groups have nearly identical RTs, showing that schizophrenic participants did not differ from normal participants with respect to pure reaction times to visual stimuli. In the following three blocks (blocks 4-6), where participants knew the sequence and received the instruction to anticipate, control participants showed a highly significant decrease in RTs with respect to schizophrenic participants (ANOVA, Table 2). The RTs in control participants dropped down to 280 and $270 \mathrm{~ms}$ in the Temporal and Spatial experiments, respectively, while the RTs for the schizophrenic group dropped only to $680 \mathrm{~ms}$ (Temporal) and $795 \mathrm{~ms}$ (Spatial). In the last, random sequence block (R), where no anticipation was possible, RTs grew for both groups, but significantly less so in schizophrenics than in controls.

\subsection{Anticipation conditions}

In the other two conditions, where participants explicitly received the sequence information at the beginning of the test, the response pattern was very similar across the two groups, though the RTs were higher for the schizophrenic participants (Fig. 4). Control participants had mean RTs between 100 and $200 \mathrm{~ms}$ and schizophrenic participants between 600 and $700 \mathrm{~ms}$, except in the easiest condition where their RTs were around $350 \mathrm{~ms}$. In both groups, RTs remained stationary during the blocks that include instructions to anticipate (blocks 1-7). The ANOVA analysis showed a highly significant difference between groups but no interaction between groups and
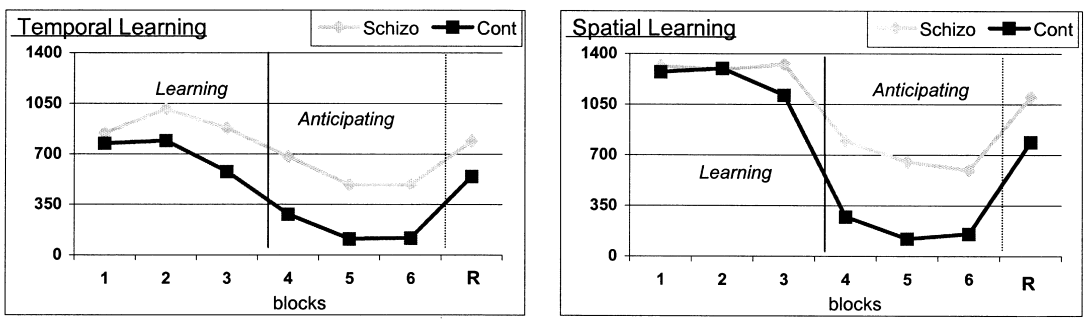

Fig. 3. RT for Schizophrenics (Schizo) and Controls (Cont) during the blocks of the learning condition of the Temporal and Spatial experiments (blocks 1-3, participants learned the sequence; blocks 3-6, participants anticipated; $R$, the block with random sequence). 
Table 2

ANOVA analysis of the RT in the three conditions of the two experiments for the two groups

\begin{tabular}{|c|c|c|c|}
\hline $\begin{array}{l}\text { ANOVA repeated } \\
\text { measures }\end{array}$ & Groups & Blocks & Groups $\times$ Blocks \\
\hline $\begin{array}{l}\text { Learning condition } \\
\text { (before anticipation) }\end{array}$ & & Blocks 1-3 & \\
\hline Temporal experiment & $\begin{array}{l}F(1,25)=1.39 \\
P=0.25\end{array}$ & $\begin{array}{l}F(2,50)=1.23 \\
P=0.3\end{array}$ & $\begin{array}{l}F(2,50)=0.71 \\
P=0.50\end{array}$ \\
\hline Spatial experiment & $\begin{array}{l}F(1,20)=1.84 \\
P=0.37\end{array}$ & $\begin{array}{l}F(2,40)=0.67 \\
P=0.52\end{array}$ & $\begin{array}{l}F(2,40)=0.73 \\
P=0.49\end{array}$ \\
\hline $\begin{array}{l}\text { Learning condition } \\
\text { (anticipation) }\end{array}$ & & Blocks 4-6 & \\
\hline Temporal experiment & $\begin{array}{l}F(1,38)=13.7 \\
P=0.0007\end{array}$ & $\begin{array}{l}F(2,76)=7.70 \\
P=0.0009\end{array}$ & $\begin{array}{l}F(2,76)=0.46 \\
P=0.96\end{array}$ \\
\hline Spatial experiment & $\begin{array}{l}F(1,36)=18.6 \\
P=0.0001\end{array}$ & $\begin{array}{l}F(2,72)=8.56 \\
P=0.0005\end{array}$ & $\begin{array}{l}F(2,72)=0.32 \\
P=0.74\end{array}$ \\
\hline $\begin{array}{l}\text { Anticipation condition } \\
\text { (Easy sequence) }\end{array}$ & & Blocks 1-7 & \\
\hline Temporal experiment & $\begin{array}{l}F(1,38)=15.4 \\
P=0.0003\end{array}$ & $\begin{array}{l}F(6,228)=4.7 \\
P=0.0002\end{array}$ & $\begin{array}{l}F(6,228)=2.4 \\
P=0.29\end{array}$ \\
\hline Spatial experiment & $\begin{array}{l}F(1,37)=19.9 \\
P<0.0001\end{array}$ & $\begin{array}{l}F(6,222)=2.1 \\
P=0.057\end{array}$ & $\begin{array}{l}F(6,222)=0.58 \\
P=0.74\end{array}$ \\
\hline $\begin{array}{l}\text { Anticipation condition } \\
\text { (Difficult sequence) }\end{array}$ & & Blocks 1-7 & \\
\hline Temporal experiment & $\begin{array}{l}F(1,38)=20.7 \\
P<0.0001\end{array}$ & $\begin{array}{l}F(6,228)=1.3 \\
P=0.018\end{array}$ & $\begin{array}{l}F(6,228)=0.29 \\
P=0.94\end{array}$ \\
\hline Spatial experiment & $\begin{array}{l}F(1,34)=28.7 \\
P<0.0001\end{array}$ & $\begin{array}{l}F(6,204)=2.6 \\
P=0.018\end{array}$ & $\begin{array}{l}F(6,204)=0.69 \\
P=0.66\end{array}$ \\
\hline
\end{tabular}

blocks (Table 2). Finally, RTs increased for the random sequence block (R). Although this increase was much more marked in the control group, the fact that the schizophrenic patients also increased their RTs for the random sequence clearly indicates that they were still presenting some degree of anticipation.

The group mean of the percentages of anticipation (e.g. the mean difference between RTs for the anticipation blocks and RTs in the random sequence block) is shown for the two experiments in Fig. 5. The group means for the percentages of anticipation for all the blocks in each condition are shown at the top; those for the best block in each condition are shown at the bottom. An individual analysis ( $t$-test, bi-varied) of the three conditions repeated in the Temporal and Spatial experiments showed a significant reduction in the capacity of anticipation in schizophrenic participants. This reduction was significant for analyses concerning the anticipation across blocks and the "best block" of anticipation (see $P$ values in the legend of Fig. 5). Control participants showed percentages of anticipation between 70 and $90 \%$ and schizophrenic participants between 30 and 50\% only. For the 'best block' analysis, percentages were between 85 and $95 \%$ in the control participants and between 50 and $65 \%$ in the schizophrenic patients. The ANOVA analysis (Table 3) revealed a difference between conditions in Temporal and Spatial experiments but no interac- 

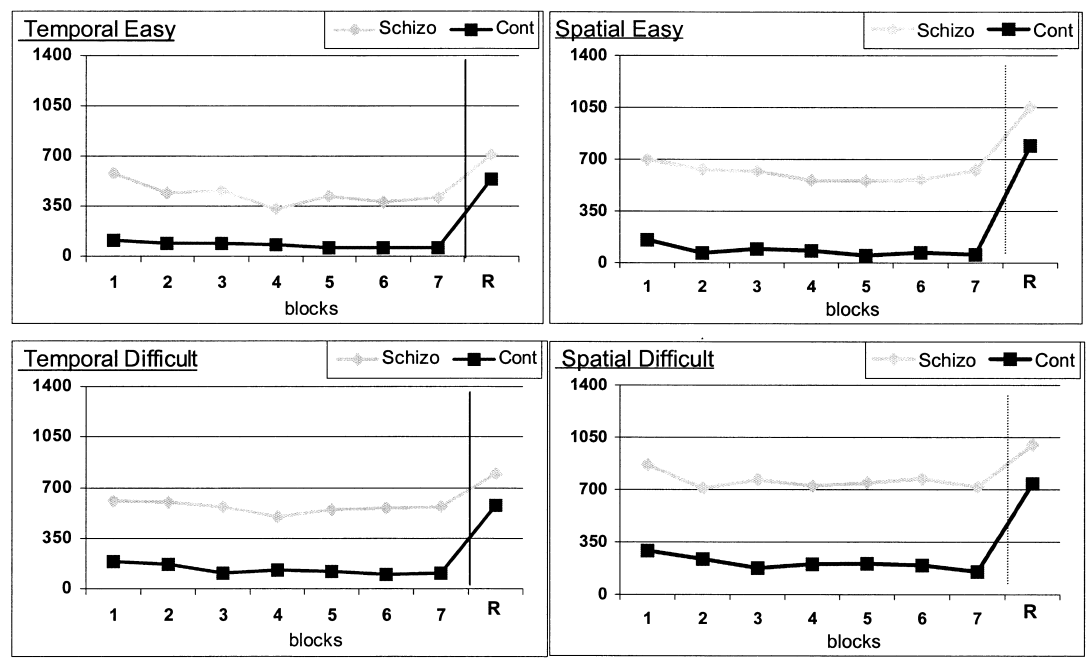

Fig. 4. RT for Schizophrenics (Schizo) and Controls (Cont) in the anticipation conditions (easy and difficult) of the Temporal and Spatial experiments (blocks $1-7$, participants anticipate; $R$, the block with random sequence).

tion between groups and conditions, neither between groups nor experiments. This result means that although the percentages of anticipation in both groups differed, they were similarly affected by the different conditions across experiments.

To measure the change in anticipation performance across blocks, the difference between the percentage of anticipation for the best block and the percentage for all the blocks was calculated. The mean reductions in the percentage of anticipation in the three conditions of the two experiments for the two groups are shown in Table 4. Schizophrenic participants presented a larger reduction in the Easy condition for the two experiments and in the Difficult condition but only for the Temporal experiment ( $t$-test). Curiously, the changes in the differences between the percentages are significant only in the control group.

Finally, we calculated the schizophrenics' scores obtained in the psychiatric Positive and Negative Scales (SAPS and SANS) with the percentage of anticipation values. No significant correlation was found (data not shown).

\subsection{Variability}

A characteristic of the studies of schizophrenic illness is the large variability between patients. To evaluate the variability of the percentage of anticipation, the participants were split into five percentage anticipation categories $(<0 \%, 0-25 \%$, $25-50 \%, 50-75 \%$ and $75-100 \%$ ). Fig. 6 shows the number of participants per category in relation to the different conditions. A large heterogeneity was observed across patients in the two analyses ('All Blocks' and 'Best Blocks'); by contrast, almost all control participants had a high percentage of anticipation scores. 

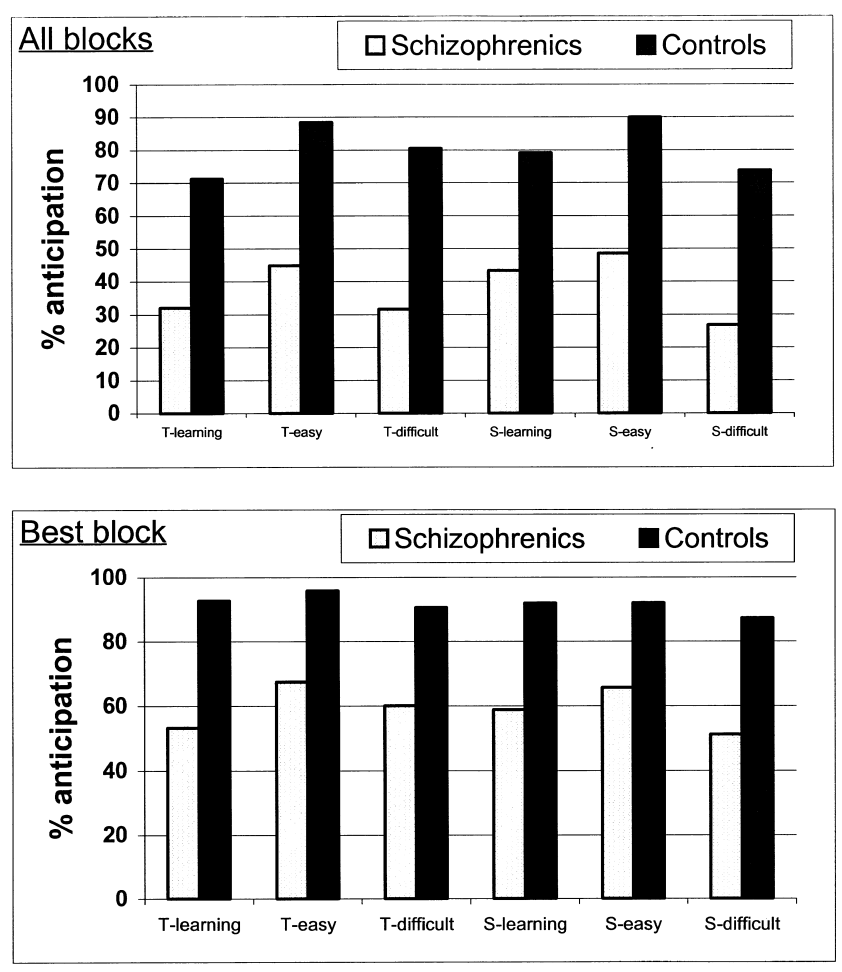

Fig. 5. Percentage of anticipation in the three conditions (learning, anticipation easy and anticipation difficult) of the two experiments ( $T=$ Temporal experiment and $S=$ Spatial experiment) for Schizophrenics and Controls. (Top) Percentage of anticipation calculated with the mean of all blocks of each condition ( $t$-test, bi-varied, left to right: $P=0.005, P=0.0001, P<0.0001, P=0.01, P=0.001$, $P=0.0001$ ). (Bottom) Percentage of anticipation calculated with the mean of the best block of each condition ( $t$-test, bi-varied, left to right: $P=0.0007, P=0.001, P=0.002, P=0.001, P=0.026$, $P=0.001)$.

\section{Discussion}

\subsection{A working memory impairment}

The above results reveal a highly specific deficit in anticipatory behavior in the group of schizophrenic patients. This deficit cannot be due to the consequence of impairments in elementary perceptual or motor functions. The patients had no difficulty performing the basic sensorimotor task of associating a color displayed on the computer screen with the corresponding color button; they also showed normal values of reaction times when they responded to the presentation of colors before knowing the sequence. The deficit in anticipating was very similar for the two types of sequences (temporal and spatial). The number of errors remained low and was not significantly different from that of control participants. In addition, all patients were able, more or less rapidly, to learn and to remember the target 
Table 3

ANOVA analysis of the percentage of anticipation in the three conditions of the two experiments for the two groups

\begin{tabular}{llll}
\hline $\begin{array}{l}\text { ANOVA repeated } \\
\text { measures }\end{array}$ & Conditions & Groups $\times$ Conditions & Groups $\times$ Experiments \\
\hline All blocks & & & \\
Temporal experiment & $F(2,76)=4.72$, & $F(2,76)=0.46$, & - \\
& $P=0.012$ & $P=0.63$ & \\
Spatial experiment & $F(2,68)=14.9$, & $F(5,68)=2.1$, & - \\
& $P<0.0001$ & $P=0.13$ & \\
Both & - & - & $F(1,38)=0.46$, \\
& & & $P=0.5$ \\
Best block & & $F(2,76)=1.44$, & - \\
Temporal experiment & $F(2,76)=3.23$, & $P=0.243$ & - \\
& $P=0.045$ & $F(2,68)=3.2$, & \\
Spatial experiment & $F(2,68)=7.4$, & $P=0.0468$ & $F(1,38)=2.1$, \\
& $P=0.0012$ & - & $P=0.16$ \\
Both & - & & \\
& & & \\
& & &
\end{tabular}

sequences in each of the experimental conditions. Although control participants typically required two blocks to acquire the sequence, patients needed three blocks. Failures (inability to acquire the sequence after seven blocks) were about three times more frequent in patients than in controls; however, those who had not been able to disclose the sequence by themselves, when properly trained by the experimenter, were able to memorize it. These results indicate that although patients learned more slowly than controls, they had retention abilities compatible with task execution.

The schizophrenic patients had major difficulties when we introduced the instruction to respond in anticipation to the colors, i.e. when they had to shift from a sensory-guided to a memory-guided type of behavior. In this condition, patients could only partly reduce their RTs in comparison with their performance prior to learning. Whereas control participants produced responses times in the range of 100-200 ms (i.e. well below typical reaction times), the schizophrenics were barely able to perform better than 600-700 ms. Note, however, that some degree of anticipation persisted in patients, as demonstrated by the fact that in all conditions their responses times were shorter than their purely reactive RTs in the random sequence blocks. Thus, the patients in this experiment, though strongly impaired in using the explicit knowledge they had available, had retained the ability to understand and to perform the task.

To anticipate an incoming event is a cognitive process which requires the knowledge of regularities in the temporal unfolding of external events. In the particular process of anticipating the next incoming color, participants must use simultaneously the notion of a repetitive sequence, the sequence information stored in memory, the instruction to anticipate, and the perception of the color of the stimulus. All these components are integrated to produce a motor representation which activates the motor command to push the correct button. This integrative process, which 
Table 4

Differences between the percentage of anticipation for the best block and the percentage of anticipation for all the blocks in the three conditions in the two experiments for the two groups

\begin{tabular}{lcccccc}
\hline & T-learning & T-easy & T-difficult & S-learning & S-easy & S-difficult \\
\hline Schizophrenics & 21.31 & 22.715 & 28.61 & 15.535 & 17.195 & 20.7 \\
Controls & 21.575 & 7.39 & 10.065 & 12.09 & 2.17 & 13.565 \\
$P$ value $(t$-test $)$ & 0.9692 & 0.0009 & 0.0002 & 0.4846 & 0.0009 & 0.1007 \\
\hline
\end{tabular}

has received considerable interest in neuropsychology, fits the definition of the 'working memory' which operates at the interface between memory, attention and perception.

Working memory has often been associated with activation of the frontal lobes. In normal subjects, fronto-polar prefrontal cortex is more active, using fMRI, when participants have to keep in mind a main goal while performing concurrent subgoals (Koechlin, Basso, Pietrini, Panzer, \& Grafman, 1999). Another recent fMRI study demonstrated frontal lobe specialization in maintaining working memory representations that integrate verbal and spatial representations (Prabhakaran, Narayanan, Zhao, \& Gabrieli, 2000). It has been proposed that working memory activates a network of interconnected cortical and subcortical areas including the prefrontal cortex and the hippocampus (Goldman-Rakic, 1987). Clinical studies in brainlesioned subjects support this dissociation between the role of amygdalo-hippocampal regions in memory and that of frontal regions in executive functions (Owen, Morris, Sahakian, Polkey, \& Robbins, 1996). In general, patients with frontal lesions
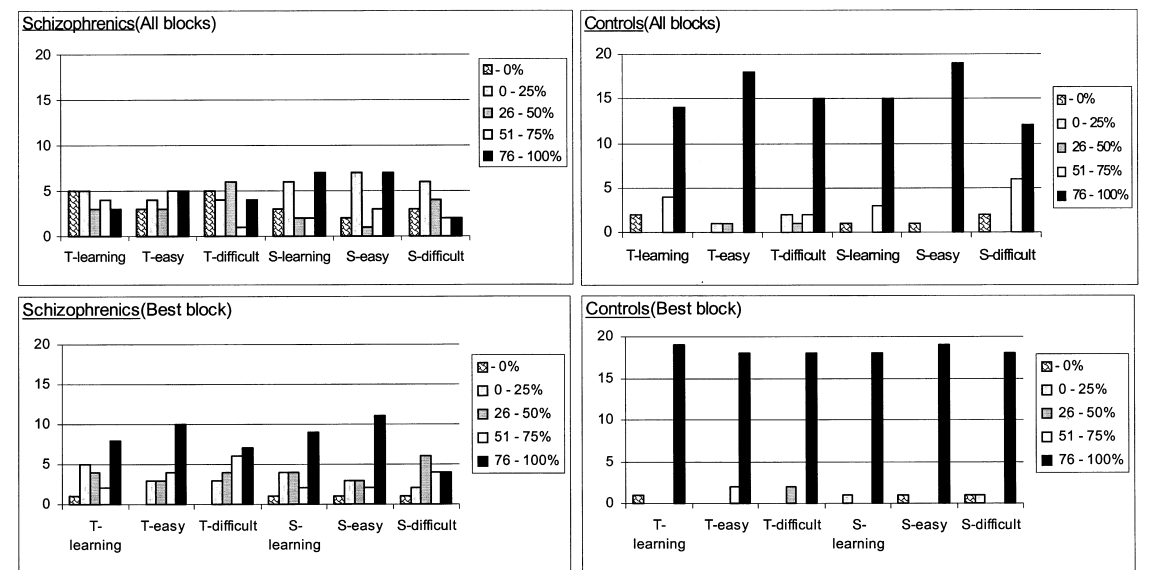

Fig. 6. Relation between the number of participants and the percentage of anticipation (divided into five groups: $<0 \%, 0-25 \%, 25-50 \%, 50-75 \%$ and $75-100 \%$ ) in the three conditions (learning, anticipation easy and anticipation difficult) of the two experiments ( $T=$ Temporal experiment and $S=$ Spatial experiment) for Schizophrenics (left) and Controls (right). In one case the percentage of anticipation was calculated with the mean of all blocks (all blocks), and in the other it was calculated with the best block (best block) of each condition. 
are typically unable to plan ahead and to organize their behavior toward the achievement of a goal (Godefroy, Cabaret, Petit-Chenal, Pruvo, \& Rousseaux, 1999). Instead, they tend to be driven by external events. Anticipation as it is understood here has not been studied in frontal patients, except in tasks involving memoryguided saccades (Walker et al., 1998): as already mentioned, responses given in these tasks are not explicitly guided and are not based on memorized knowledge.

A deficient functional role of frontal cortex might also explain the problems met by schizophrenic patients during working memory tasks (Carter et al., 1998), and specifically in our anticipation paradigm. In our experiments, schizophrenic patients did not show any difficulty in memorizing the sequence information. Their performance decreased when they needed to guide their movements by a mental representation which had to be refreshed at each trial. This representation is constructed mostly with memory elements because sensory cues appear only later. The patients' deficit was not with the instructions of the task either because they made the same number of errors as controls. Hypofrontality is a common finding in neuroimaging studies of schizophrenic patients. It has been suggested that hypofrontality might be task-dependent (Curtis et al., 1999). In a PET study by Spence, Hirsch, Brooks, and Grasby (1998), schizophrenic patients with delusions and hallucinations were scanned while they performed a free selection of joystick movements (a working memory demanding task). The patients showed significantly less activation in their left dorsolateral prefrontal cortex than control participants. This difference was greatly attenuated in the same patients when their symptoms remitted under symptomatic treatment. This study illustrates the fact that hypofrontality is a dynamic phenomenon across time.

Finally, the fact that all patients tested were under treatment during the experiment cannot represent a likely explanation for their deficit in working memory. Although antipsychotic drugs (like haloperidol, for example) can deteriorate working memory in monkeys, their effect has been shown to be the opposite in schizophrenic patients: they generally enhance cognitive performances in addition to decreasing clinical symptoms (Purdon et al., 2000).

\subsection{The behavioral and clinical consequences of lack of anticipation}

A deficit in using available information to correctly anticipate an incoming event likely represents an explanatory framework for some of the pathological aspects of schizophrenic behavior in relation to possible frontal hypoactivation. Such a deficit may be particularly deleterious in the domain of action, where a lack of anticipation may create situations where the consequences of actions are not properly evaluated. When planning (or intending) to move his/her hand to the right, for example, a normal individual anticipates to see and feel it moving in that direction. If the anticipation process is impaired, the direction in which the hand is seen to move may appear to be unrelated to the desired direction. This might be the sort of situation that schizophrenic patients are faced with: in the above example, the patient may feel that his/her hand was displaced by an external agent, or even that it was an alien hand. When asked whom this hand belongs to, or who was 
the author of this movement, the patient may adopt different strategies: he may attribute the hand and/or the movement to an external agent, or alternatively he may use a default strategy by attributing the movement to himself. Both strategies correspond to frequently observed clinical symptoms (delusion of influence) in schizophrenic patients. In previous studies we have repeatedly shown that such patients tend to over-attribute to themselves movements performed by other agents (Daprati et al., 1997) and have increased thresholds for detecting movements differing from those they have actually performed (Franck et al., 2001). Lack of anticipation would thus preclude the normal match of actions with their internal representations, with the consequence that self-performed actions would not be recognized and would be misattributed. This hypothesis appears to be complementary with that of Frith, Blakemore, and Wolpert (2000). These authors postulate that experience of alien control arises from a lack of awareness of the predicted limb position when a movement is to be performed. Our present data provide support for this idea by directly demonstrating the cause of this lack of awareness.

Finally, it is tempting to generalize this reasoning to other aspects of symptomatology in schizophrenia. Indeed, abnormal time integration was proposed decades ago to explain the production of schizophrenic symptoms (Minkowski, 1927). Impairment of patients in matching actions and representations of actions could lead to the production of positive symptoms, such as incongruous actions or hallucinations, as well as to negative symptoms like an impossibility to act, apathy, or lack of social interactions. Anticipation is clearly involved in social communication, for monitoring one's own mental states and for understanding and evaluating the responses of other individuals.

\section{Acknowledgements}

We thank Professor M. Marie-Cardine for hosting our experiments in his psychiatric department, the clinical staff for its collaboration, and B. Messaoudi for his technical support with the apparatus. Luis Murillo and Ira Noveck are gratefully acknowledged for their valuable comments.

\section{References}

Aleman, A., Hijman, R., de Haan, E. H., \& Kahn, R. S. (1999). Memory impairment in schizophrenia: a meta-analysis. American Journal of Psychiatry, 156 (9), 1358-1366.

Andreasen, N. C. (1983). The scale for the assessment of negative symptoms (SANS), Iowa City, IA: The University of Iowa.

Andreasen, N. C. (1984). The scale for the assessment of positive symptoms (SAPS), Iowa City, IA: The University of Iowa.

Baddeley, A. (1998). Recent developments in working memory. Current Opinion in Neurobiology, 8 , 234-238.

Bernard, D., Lancon, C., \& Bougerol, T. (1997). Attention models in evaluating schizophrenia. Encephale, 23 (2), 113-118.

Carter, C. S., Perlstein, W., Ganguli, R., Brar, J., Mintun, M., \& Cohen, J. D. (1998). Functional hypo- 
frontality and working memory dysfunction in schizophrenia. American Journal of Psychiatry, 155 (9), 1285-1287.

Crawford, T. J., Haeger, B., Kennard, C., Reveley, M. A., \& Henderson, L. (1995). Saccadic abnormalities in psychotic patients. I. Neuroleptic-free psychotic patients. Psychological Medicine, 25, 461-471.

Curtis, V. A., Bullmore, E. T., Morris, R. G., Brammer, M. J., Williams, S. C., Simmons, A., Sharma, T., Murray, R. M., \& McGuire, P. K. (1999). Attenuated frontal activation in schizophrenia may be task dependent. Schizophrenia Research, 37 (1), 35-44.

Danion, J. M., Rizzo, L., \& Bruant, A. (1999). Functional mechanisms underlying impaired recognition memory and conscious awareness in patients with schizophrenia. Archives of General Psychiatry, 56 (7), 639-644.

Daprati, E., Franck, N., Georgieff, N., Proust, J., Pacherie, E., Dalery, J., \& Jeannerod, M. (1997). Looking for the agent: an investigation into consciousness of action and self-consciousness in schizophrenic patients. Cognition, 65, 71-86.

Dominey, P., \& Georgieff, N. (1997). Schizophrenics learn surface but not abstract structure in a serial reaction time task. NeuroReport, 8, 2877-2882.

Fleming, K., Goldberg, T. E., Binks, S., Randolph, C., Gold, J. M., \& Weinberger, D. R. (1997). Visuospatial working memory in patients with schizophrenia. Biological Psychiatry, 41, 43-49.

Franck, N., Farrer, C., Georgieff, N., Marie-Cardine, M., Daléry, J., d'Amato, T., \& Jeannerod, M. (2001). Defective recognition of one's own actions in schizophrenic patients. American Journal of Psychiatry, $158,454-459$.

Frith, C. D., Blakemore, S., \& Wolpert, D. M. (2000). Explaining the symptoms of schizophrenia: abnormalities in the awareness of action. Brain Research Reviews, 31, 357-363.

Godefroy, O., Cabaret, M., Petit-Chenal, V., Pruvo, J. P., \& Rousseaux, M. (1999). Control functions of the frontal lobes. Modularity of the central-supervisory system? Cortex, 35 (1), 1-20.

Goldman-Rakic, P. S. (1987). Circuitry of primate prefrontal cortex and regulation of behavior by representational memory. In F. Plum (Ed.), Higher functions of the brain. Handbook of physiology. The nervous system (Section I, Vol. V, pp. 373-417). Bethesda, MD: American Physiological Society.

Goldman-Rakic, P. S. (1994). Working memory dysfunction in schizophrenia. Journal of Neuropsychiatry \& Clinical Neurosciences, 6 (4), 348-357.

Grapperon, J., \& Delage, M. (1999). Stroop test and schizophrenia. Encephale, 25, 50-58.

Koechlin, E., Basso, G., Pietrini, P., Panzer, S., \& Grafman, J. (1999). The role of the anterior prefrontal cortex in human cognition. Nature, 399, 148-151.

Kuperberg, G., \& Heckers, S. (2000). Schizophrenia and cognitive function. Current Opinion in Neurobiology, 10 (2), 205-210.

Kuperberg, G. R., McGuire, P. K., \& David, A. S. (1998). Reduced sensitivity to linguistic context in schizophrenic thought disorder: evidence from on-line monitoring for words in linguistically anomalous sentences. Journal of Abnormal Psychology, 107 (3), 423-434.

Laws, K. R. (1999). A meta analytic review of Wisconsin card sort studies in schizophrenia: general intellectual deficit in disguise? Cognitive Neuropsychiatry, 4 (1), 1-35.

Minkowski, E. (1927). La schizophrénie. Psychopathologie des schizoÏdes et des schizophrènes. Editions Payot \& Rivages, Paris: Payot.

Nuechterlein, K. H., \& Dawson, M. E. (1984). Information processing and attentional functioning in the developmental course of schizophrenic disorders. Schizophrenia Bulletin, 10 (2), 160-203.

Owen, A. M., Morris, R. G., Sahakian, B. J., Polkey, C. E., \& Robbins, T. W. (1996). Double dissociations of memory and executive functions in working memory tasks following frontal lobe excisions, temporal lobe excisions or amygdalo-hippocampectomy in man. Brain, 119, 1597-1615.

Pallier, C., Dupoux, E., \& Jeannin, X. (1997). Expe: an expandable programming language for psychological experiments. Behavior Research Methods, Instruments and Computers, 29 (3), 322-327.

Park, S., Holzman, P. S., \& Goldman-Rakic, P. S. (1995). Spatial working memory deficits in the relatives of schizophrenic patients. Archives of General Psychiatry, 52 (10), 821-828.

Prabhakaran, V., Narayanan, K., Zhao, Z., \& Gabrieli, D. E. (2000). Integration of diverse information in working memory within the frontal lobe. Nature Neuroscience, 3, 85-89.

Purdon, S. E., Jones, B. D., Stip, E., Labelle, A., Addington, D., David, S. R., Breier, A., \& Tollefson, G. 
D. (2000). Neuropsychological change in early phase schizophrenia during 12 months of treatment with olanzapine, risperidone, or haloperidol. Archives of General Psychiatry, 57 (3), 249-258.

Servan-Schreiber, D., Cohen, J. D., \& Steingard, S. (1996). Schizophrenic deficits in the processing of context. Archives of General Psychiatry, 53, 1105-1112.

Spence, S. A., Hirsch, S. R., Brooks, D. J., \& Grasby, P. M. (1998). Prefrontal cortex activity in people with schizophrenia and control subjects. Evidence from positron emission tomography for remission of 'hypofrontality' with recovery from acute schizophrenia. British Journal of Psychiatry, 172, 316323.

Walker, R., Husain, M., Hodgson, T. L., Harrison, J., \& Kennard, C. (1998). Saccadic eye movement and working memory deficits following damage to human prefrontal cortex. Neuropsychologia, 36 (11), 1141-1159.

Wexler, B. E., Stevens, A. A., Bowers, A. A., Sernyak, M. J., \& Goldman-Rakic, P. S. (1998). Word and tone working memory deficits in schizophrenia. Archives of General Psychiatry, 55 (12), 1093-1096. 